\title{
Mobile Access and Adoption of the Swedish National Patient Portal
}

\author{
Maria HÄGGLUND ${ }^{\mathrm{a}, 1}$, Charlotte BLEASE ${ }^{\mathrm{b}}$ and Isabella SCANDURRA ${ }^{\mathrm{c}}$ \\ 'Department of Women's and Children's Health, Uppsala university, Uppsala, Sweden \\ ${ }^{b}$ Harvard Medical School and Beth Israel Deaconess Medical Center, Boston, USA \\ ${ }^{c}$ Informatics, School of Business, Örebro University, Örebro, Sweden
}

\begin{abstract}
Patient portals are used as a means to facilitate communication, performing administrative tasks, or accessing one's health record. In a retrospective analysis of real-world data from the Swedish National Patient Portal 1177.se, we describe the rate of adoption over time, as well as how patterns of device usage have changed over time. In Jan 2013, 53\% of all visits were made from a computer, and $38 \%$ from a mobile phone. By June $2020,77 \%$ of all visits were made from a mobile phone and only $20 \%$ from a computer. These results underline the importance of designing responsive patient portals that allow patients to use any device without losing functionality or usability.
\end{abstract}

Keywords. Patient portals, adoption, mobile access,

\section{Introduction}

Patient portals are used to facilitate communication between patients and healthcare professionals, as well as for performing administrative tasks, such as appointment bookings and prescription renewals [1]. Patients are also increasingly provided with access to their electronic health records (EHRs) [2], sometimes referred to as patient accessible EHRs (PAEHRs) [3] [4] or open notes [5], through portals. Patient portals have been widely implemented, yet adoption often remains low [1].

In Sweden, a national patient portal is used that connects to all EHR systems used in the 21 regions (who are responsible for providing healthcare) [4], through a national health information exchange platform [6][7]. Authentication with a national e-ID gives access to a number of administrative services as well as the PAEHR Journalen. Although the regions are autonomous and can prioritize which eHealth services to focus on, the national eHealth strategy stipulates that there should be only one online healthcare access point for patients [8]. Thus, a national patient portal ' $1177 . \mathrm{se}$ ' is available for everyone seeking healthcare or health-related information in Sweden, consisting of three parts;

1. 1177 on the phone - a telephone advice service reached through the national phone number 1177 ,

2. 1177.se on the web - without authentication the public can access and search among information about illnesses, symptoms and treatments, as well as information about healthcare in the region. The portal is national, but each

${ }^{1}$ Corresponding Author, Maria Hägglund, Department of Women's and Children's Health, Uppsala University, Dag Hammarskjölds väg 14B, 75237 Uppsala, Sweden. Email: maria.hagglund@kbh.uu.se. 
region in Sweden can adapt the information to its inhabitants, and users can switch between regions,

3. 1177.se personal eHealth services - after authentication (using a nationally approved e-ID) individuals have access to personalized e-services where they can e.g. send secure messages, request, reschedule or cancel appointments, renew prescriptions and access documents such as sick-leave. Functionality can vary based on region/healthcare provider.

Similar national patient portals are implemented throughout the Nordic countries, and healthcare provider specific portals are common beyond the Nordic context. Yet, implementation does not equal adoption, and in order to reap the expected benefits, we have to increase our knowledge about how patients access and use patient portals in order to better adapt them to users' needs. A key issue in this is what devices patients prefer to use. The aim of this paper is therefore to analyze and describe how patients access the Swedish national patient portal and how adoption has evolved over time.

\section{Methods}

A retrospective analysis of real world data on the use and adoption of the National Patient Portal has been performed. De-identified and aggregated usage data from the National Patient Portal and associated eHealth services are provided online by Inera AB [9], and is used for this study.

Adoption is assessed by analyzing the number of visits to the National Patient Portal from Jan 2013 until June 2020, as this is the data that is provided as open access. The open pages of the National Patient Portal (1177 on the web) does not require log-in, and therefore it is not possible to keep track of demographic data on the users. Therefore we also include data on the number of users of the personal eHealth services on 1177.se, although it may not be representative to all users of 1177.se.

Data on what device (computer, mobile phone, or tablet) a patient use to access the open pages of 1177.se is also presented, to highlight how access to the National Patient Portal has evolved over time from 2013 to 2020 . This is closely related to Internet access and usage among the Swedish population overall.

\subsection{Internet use among the Swedish population}

Internet usage is high in Sweden, and according to the most recent survey "the Swedes and the Internet" from the Swedish Internet Foundation, 95\% of the Swedish population use the internet and $91 \%$ uses the internet on a daily basis [10]. Both computers and mobile phones are frequently used to access the internet ( $91 \%$ and $90 \%$ respectively). $53 \%$ of the respondents also use digital services to manage their healthcare, e.g. book appointments, check lab results etc.

\section{Results}

Late 2019, Sweden had approximately 10.3 million inhabitants, compared to 9.6 million in 2013 , an increase with $7 \%$. The open pages of the national patient portal $1177 \mathrm{had}$ 3210189 visits in January 2013, whereas the monthly number of visits in January 2020 
was 13325793 (an increase of over 400\%). Figure 1 shows the development of monthly visits to 1177 from Jan 2013 to June 2020. Dips in the curve occurs during the summer months (June-Aug) every year, whereas visits peak in Jan-March, coinciding with the Swedish flu season. In 2020, the COVID-19 pandemic created an outstanding peak of visits in March with 18268469 visits.

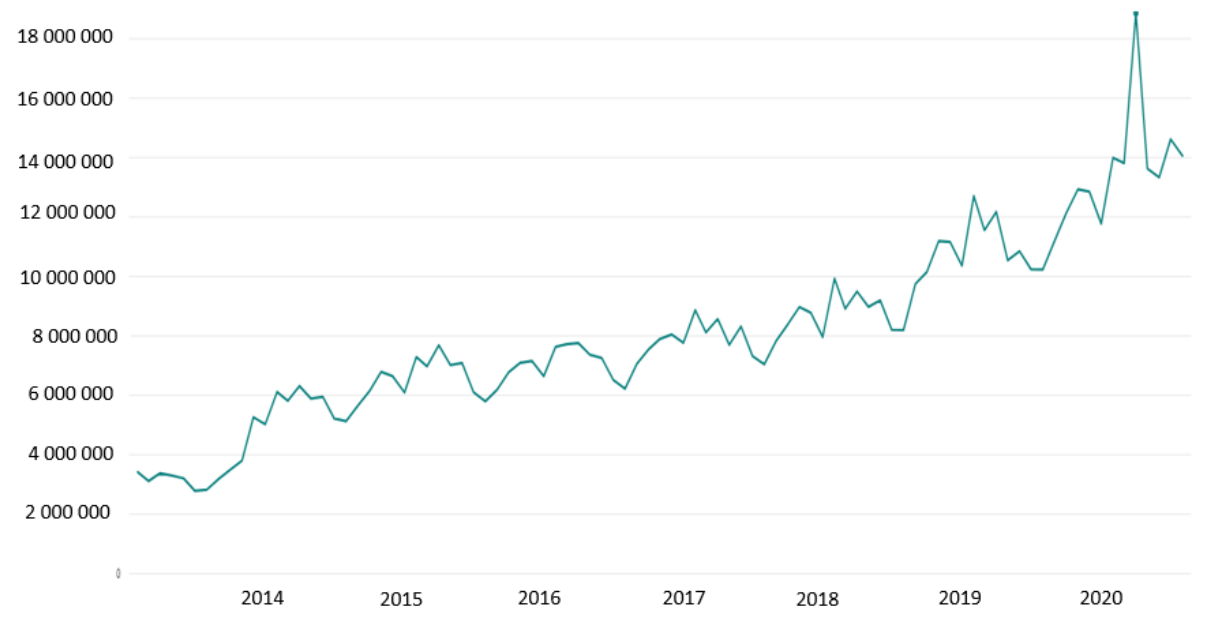

Figure 1. Number of monthly visits to 1177.se January 2013 - June 2020.

The personal eHealth services provided on the National Patient Portal require for an individual to sign in to their account. Therefore, it is also possible to keep track of how many individuals access these services. In June 2020, 7230063 persons had accessed their personal eHealth services at least once. These services have also seen a dramatic increase in use; in Jan 2013 a total of 29228 logins were made to the portal, made by 17572 unique users, and the corresponding numbers in June 2020 was 7189878 log ins made by 1724735 unique users. Number of log-ins follows a similar pattern of dropping in the summer months and increasing in winter, however 2020 stands out with an increase in log-ins during June. This is likely due to an increase in COVID-19 testing (both for the virus and for antibodies), where patients use the national patient portal to both book appointments and to access their test results.

In addition to the increase in visits to the National Patient Portal, a possibly more striking change has occurred in how people access the website. In January 2013, 1716175 visits were on a computer (53\% of all visits), 1210561 from a mobile phone $(38 \%)$ and 278635 from a tablet (9\%) (Figure 2).

In June 2020, mobile phones dominates as the most common device for visiting the National Patient Portal (Figure 2); computers 2805176 (20\%), mobile phones $11001189(77 \%)$, and tablets $468227(3 \%)$. This is however not due to a decrease in visits from computers and tablets, since the number of visits from these devices has increased over time; rather, the proportion of mobile phone visits has increased greatly during this period. 

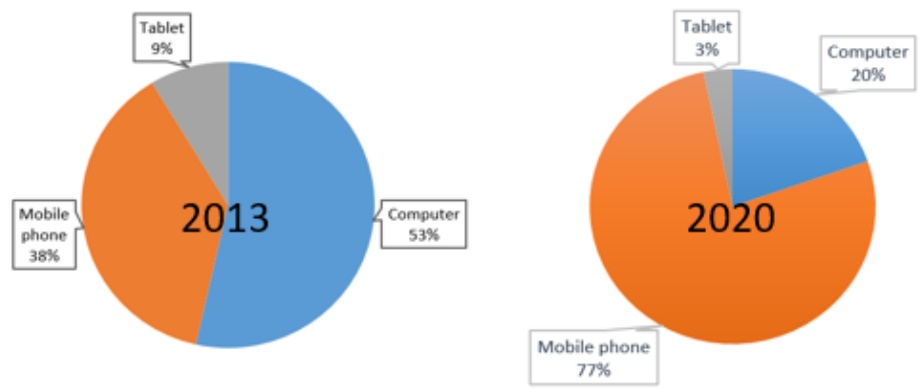

Figure 2. Devices used to access 1177.se January 2013 and June 2020 respectively.

The increase in visits from mobile phones is perhaps even clearer if we look at a graph showing development over time (Figure 3 ). Where access from a computer or tablet remains on a slow increase, the visits from mobile phones starts to increase more rapidly in 2014 and even further in 2018.

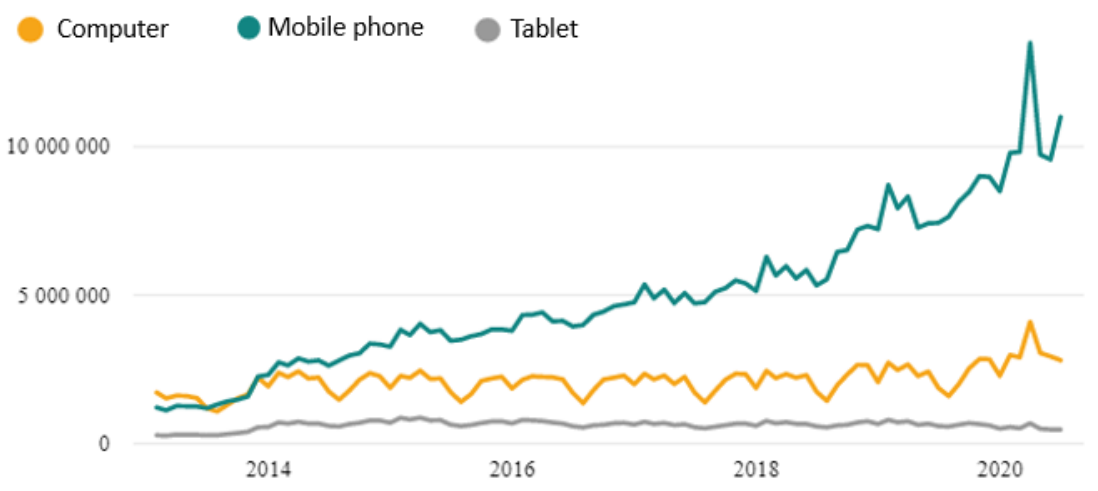

Figure 3. Monthly visits to 1177.se from different devices Jan 2013 - June 2020.

\section{Discussion and Conclusions}

Adoption takes time. In this analysis, we have looked at real-world usage data captured over 7 years of use of the Swedish National Patient Portal. From a modest start, the numbers have steadily increased every year. A recurring pattern of high usage during the winter months and lower during summer can also be seen. Adoption does not seem to have reached a plateau yet in Sweden, rather the 2020 COVID-19 pandemic seem to have further increased use of both the open and the personal eHealth services on the platform. This increase may be temporary, but it could also indicate that new user groups are finding their way to the National Patient Platform, and it will be of interest to follow-up over time. 
As indicated by the data presented in this analysis, mobile phones have become the far most common way to access the National Patient Platform. Still, computers make up for $20 \%$ of the visits, and it remains unknown whether users choose their device depending on which type of information or task they are looking to perform. Responsive design is key to ensure that different devices can be used based on the users' needs or preferences in different contexts and for different purposes. Further research is needed to deepen our understanding of when users choose a specific device over another; what are their preferences for usage when they go online e.g. accessing electronic health records, and how does mobile access affect adoption and use of eHealth services?

Another area that is important to explore further relates to socio-economic differences in accessing the National Patient Portal. Does the increase in mobile phone access correspond to new populations gaining access to the portal? Similarly, do patterns of mobile access correlate with a lack of broadband access among some patient populations? Might limitations associated with mobile phone data restrict frequency of portal usage? The analysis presented in this paper provides a starting point to continue exploring further questions relating to patient portal adoption and use.

\section{Acknowledgement}

FORTE - the Swedish Research Council for Health, Working Life and Welfare funds the research project "PACESS" (2016-00623). This research was also supported by Uppsala MedTech Science \& Innovation (https://medtech.uu.se/), a joint strategic initiative between Uppsala University and Uppsala University Hospital.

\section{References}

[1] Fraccaro P, Vigo M, Balatsoukas P, Buchan IE, Peek N, van der Veer SN. Patient Portal Adoption Rates: A Systematic Literature Review and Meta-Analysis. Stud. Health Technol. Inform. 2017;245:79-83.

[2] Hägglund M, DesRoches C, Petersen C, Scandurra I. Patients' access to health records. BMJ. 2019 Oct; 367:15725.

[3] Moll J, Rexhepi H, Cajander Å, Grünloh C, Huvila I, Hägglund M, Myreteg G, Scandurra I, Åhlfeldt RM. Patients' Experiences of Accessing Their Electronic Health Records: National Patient Survey in Sweden. J Med Internet Res. 2018; 20(11): e278.

[4] Hägglund M, Scandurra I. Patients' Online Access to Electronic Health Records: Current Status and Experiences from the Implementation in Sweden. Stud Health Technol Inf. 2017;245: 723-727.

[5] Walker J, Leveille S, Bell S, Chimowitz H, Dong Z, Elmore JG, Fernandez L, Fossa A, Gerard M, Fitzgerald P, Harcourt K, Jackson S, Payne TH, Perez J, Shucard H, Stametz R, DesRoches C, Delbanco T. OpenNotes After 7 Years: Patient Experiences With Ongoing Access to Their Clinicians' Outpatient Visit Notes. J Med Internet Res. 2019; 21(5):e13876.

[6] Davoody N, Koch S, Krakau I, Hägglund M. Accessing and sharing health information for postdischarge stroke care through a national health information exchange platform - a case study. BMC Med. Inform. Decis. Mak. 2019; 19(1):95.

[7] Sellberg N, Eltes J. The Swedish Patient Portal and Its Relation to the National Reference Architecture and the Overall eHealth Infrastructure, in Information Infrastructures within European Health Care. Springer, 2017, pp. 225-244.

[8] Ministry of Health \& Social Affairs., National eHealth - the Strategy for Accessible and Secure Information in Health and Social Care, 2010.

[9] Inera, "Inera Statistics." [Online]. Available: https://www.inera.se/aktuellt/statistik/ .

[10] Internetstiftelsen, "Svenskarna och internet 2019," 2019. 